\title{
Low-temperature electronic transport and the Coulomb blockade in oxidized films of bismuth
}

\author{
J.L. Cohn ${ }^{1}$, E. Ben-Jacob and C. Uher \\ Physics Department, University of Michigan, Ann Arbor, MI 48109, USA
}

Received 26 March 1990; accepted for publication 3 June 1990

Communicated by J.I. Budnick

\begin{abstract}
We present low-temperature $(0.2<T<10 \mathrm{~K})$ transport measurements on semicontinuous films of bismuth which form a network of coupled Bi grains. By sequentially oxidizing such films we have observed a change in the character of transport that may be associated with the emergence of a finite charging energy and Coulomb blockade for single-electron tunneling between grains. This transition is manifested most dramatically as a divergence of the thermoelectric power with decreasing temperature. Magnetoresistance measurements indicate that the crossover in the thermopower occurs abruptly when the average oxide barrier resistance exceeds a fundamental value near $h / e^{2}$.
\end{abstract}

Granular metal films have been the focus of considerable attention for the past decade in the study of the metal-insulator transition [1]. These systems generally consist of metallic grains separated by insulating barriers, the electronic coupling through which governs the character of the low-temperature resistance [2]. The limits of strong and weak coupling are well documented. In the former case the barriers have negligible activation energy and the lowtemperature resistance exhibits small quantum corrections due to weak localization [3] and Coulomb interaction [4] effects. In the weak-coupling limit electrons are localized to individual grains and the resistance depends exponentially on temperature $[5,6]$. Not understood as well is the physics at the crossover between these regimes, where neither weak nor strong localization theories are appropriate and where transport properties other than the temperature dependence of resistance [7] have rarely been addressed.

In this Letter we report low-temperature measurements of resistance, magnetoresistance and thermoelectric power in semicontinuous bismuth films. The coupling between grains is controlled by se-

Present address: Naval Research Laboratories, Washington, DC 20375, USA. quentially oxidizing individual films. Our focus is on the crossover regime where we observe a new and striking behavior of the thermopower. We propose a possible explanation based on the Coulomb blockade in small-capacitance tunnel junctions. Magnetoresistance data suggest that a sharp charging energy onset occurs when the average intergrain tunnel resistance exceeds $\sim \hbar / e^{2}$.

Samples were prepared by vapor deposition of $99.9999 \%$ purity $\mathrm{Bi}$ onto room temperature glass substrates in an oxygen atmosphere of $50 \mathrm{mTorr}$. The films were deposited in a four-probe bridge pattern $\left(0.8 \times 10 \mathrm{~mm}^{2}\right)$ with nominal thickness $55-70 \AA$ as determined by a quartz crystal monitor. A $300 \AA \mathrm{SiO}$ layer was then deposited over the conduction channel, allowing for slower, controllable oxidation. Films prepared in this fashion had post-deposition (dc) sheet resistances, $R_{\square}$, of $<1 \mathrm{k} \Omega$. Transmission electron and scanning tunneling microscopies indicate $[8,9]$ that the films are composed of closely packed crystalline Bi grains which form a multiply connected, filamentary structure. The grains are irregularly shaped with diameters ranging from 200 to $1000 \AA$. Exposing the films to air at room temperature promotes the growth of insulating $\mathrm{Bi}_{2} \mathrm{O}_{3}$ at the granular surfaces. The corresponding increases in $R_{\square}$ to values $>100 \mathrm{k} \Omega$ are associated with a reduced in- 
tergrain coupling and the development of a percolative structure, as discussed in detail elsewhere [8]. Experiments were performed in a dilution refrigerator with a superconducting solenoid providing magnetic fields perpendicular to the substrates. Steady-state thermopower measurements employed two germanium resistance sensors thermally anchored to the back of the substrates. Seebeck probes were superconducting $\mathrm{NbTi}$ wire, soldered to the film with indium. Thermoelectric voltages were monitored on a nanovoltmeter with analog output to a chart recorder.

Fig. 1 shows the typical low-temperature resistance behavior for one of our films. For $R_{\square}(4.2$ $\mathrm{K}) \ll 8 \mathrm{k} \Omega$ the resistance increases as $\ln T$, with a slope that agrees with weak disorder theories for twodimensional systems $[3,4,10]$ and is consistent with previous studies of thin, homogeneous Bi films [11]. The temperature dependence for $R_{\square}(4.2 \mathrm{~K})>8 \mathrm{k} \Omega$ is faster than $\ln T$ (approximately $T^{-1 / 2}$ ), but not yet exponential. For example, forcing a fit to the strong localization form $[5,6], R \propto \exp \left[\left(T_{0} / T\right)^{1 / 2}\right]$, yields $T_{0}<20 \mathrm{mK}$. Such a low value for $T_{0}$ is not consistent with the condition $T \gg T_{0}$ for which this expression applies.

The thermoelectric power, $S$, for the same film from fig. 1 is shown in fig. 2. Similar thermopower data have been observed in three separate films, each

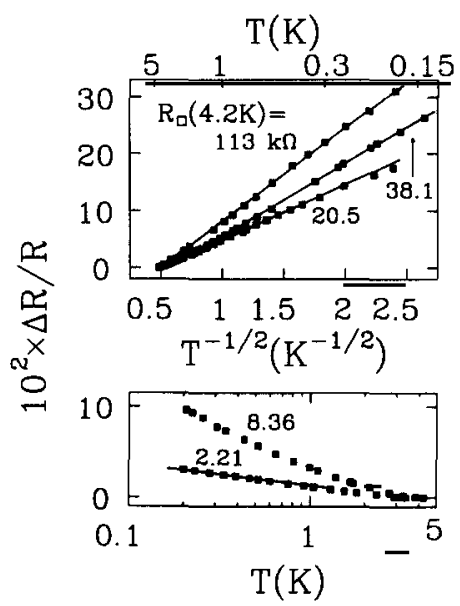

Fig. 1. Relative resistance increase, $[R(T)-R(4.2 \mathrm{~K})] / R(4.2$ $\mathrm{K})$, versus temperature for a single Bi film oxidized in steps. Each curve is labelled by the value of $R_{\square}(4.2 \mathrm{~K})$.

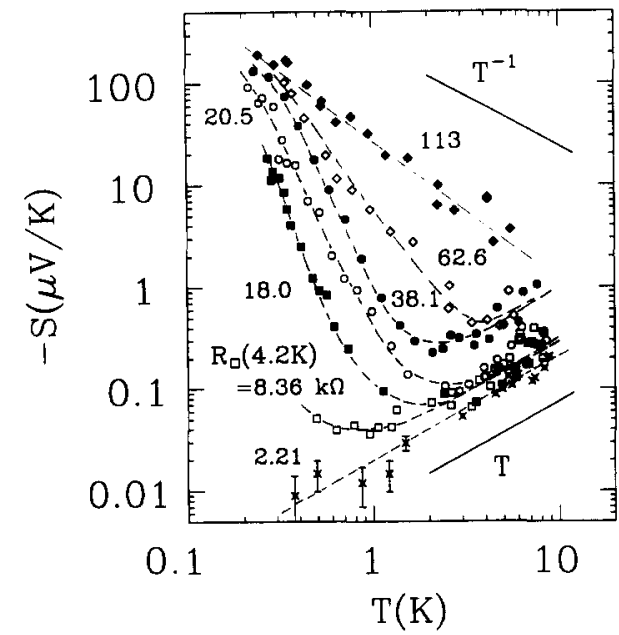

Fig. 2. Thermoelectric power versus temperature for the same film for which resistance data is shown in fig. 1. Dashed lines are guides to the eye.

oxidized in steps to span the range $1<R_{\square}<100 \mathrm{k} \Omega$. For disordered thin films, phonon drag effects are rendered negligible and we interpret the data as reflecting the carrier diffusion thermopower. The negative sign indicates a dominant contribution from electrons. The thermopower of metals [12] is expected to decrease linearly with temperature, and this behavior is observed down to the lowest temperatures for $R_{\square}(4.2 \mathrm{~K}) \ll 8 \mathrm{k} \Omega$. For $R_{\square}(4.2 \mathrm{~K})>8 \mathrm{k} \Omega$ this $T$-linear dependence gives way to a dramatic transition where $S$ diverges with decreasing temperature to values that are several orders of magnitude larger than those for samples having low $R_{\square}$. Note that the temperature at which the thermopower diverges increases with increasing oxidation and the divergence becomes less rapid, tending toward an $S \propto 1 / T$ dependence. The latter behavior is characteristic of a thermally activated process, as in semiconductors [13] where the energy gap, $E_{\mathrm{g}}$, gives rise to a low-temperature $\left(k_{\mathrm{B}} T<E_{\mathrm{g}}\right)$ thermopower, $S \propto E_{\mathrm{g}} / 2 k_{\mathrm{B}} T$. Burns et al. [14] observed a similar crossover from metallic to activated $(1 / T)$ thermopower in thin, disordered Au and Au-Pd films. The surprising feature in our data is the more rapidly diverging thermopower that characterizes films with intermediate oxidation.

Developments $[15,16]$ in the physics of small-capacitance tunnel junctions may offer insight into the 
thermopower behavior of our films. Central to a semiclassical description [15] of the current and voltage response for a single junction of capacitance $C$, is the relationship between the charging energy or Coulomb blockade, $E_{\mathrm{C}}=e^{2} / 2 C$, and the tunnel resistance, $R_{\mathrm{t}}$. This is most clearly illustrated for a weakly coupled junction, characterized by $R_{\mathrm{t}} \gg \hbar / e^{2}$ $(\approx 4.12 \mathrm{k} \Omega$ ). This resistance criterion [17] is equivalent to having localized electronic states on either side of the barrier. In this case the charge on the junction may be treated classically such that the capacitance, and hence $E_{\mathrm{C}}$, have their usual geometric meaning. A fundamental issue is how this description should be modified when the weak coupling condition is relaxed. A theoretical treatment of this problem [18] for a single junction predicts that, as the temperature and capacitance tend to zero, an abrupt breakdown of the Coulomb barrier is possible when $R_{\mathrm{t}} \sim \hbar / e^{2}$. This issue is addressed in a recent experimental study [19] of lithographically patterned single junctions and arrays, where experiments suggest that the relevant charging energy for transport may decrease below its geometric value for $R_{\mathrm{t}}$ lower than about $10 \mathrm{k} \Omega$.

For the arrangement of grains in our films we estimate $\# 1$ an average geometric capacitance on the order of $C=5 \times 10^{-16} \mathrm{~F}$, which corresponds to a charging energy $E_{\mathrm{C}} / k_{\mathrm{B}} \approx 2 \mathrm{~K}$. From the slope of the data for the highest level of oxidation in fig. 2 we find an activation energy of $\sim 1 \mathrm{~K}$, which compares favorably with our estimate of $E_{\mathrm{C}}$. This suggests that in the limit of high oxidation the charging energy is close to its geometric value with the thermopower taking on a simple activated form, $S \propto E_{\mathrm{C}} / k_{\mathrm{B}} T$. This $1 / T$ behavior is expected for discontinuous metal films [20] when transport is by thermally activated tunneling between islands. We have recently calculated [21] the thermopower for a single tunnel junction, using a semiclassical approach [15]. In this model the charging energy is included as a shift in the chemical potential of the electrode into which an

\#1 We estimate an average capacitance using the expression (see, e.g. ref. [2] ) $C=\epsilon r(1+r / 2 t)$, where $r$ is the average grain radius, $t$ is the average barrier thickness, and $\epsilon$ is the dielectric constant of the barrier material. Here we use $r \approx 250 \AA, t \approx 20$ $\AA$, and $\epsilon\left(\mathrm{Bi}_{2} \mathrm{O}_{3}\right) \approx 25$. electron tunnels. Under certain conditions ${ }^{\# 2}$ we find a thermopower that crosses over, with decreasing temperature, from a metallic ( $T$-linear) to activated $\left(\propto E_{\mathrm{C}} / k_{\mathrm{B}} T\right)$ form. A faster than $1 / T$ divergence is not predicted by these calculations.

It is possible that the sharp upturn in the thermopower for intermediate oxidation is associated with the fact that the films form an array of coupled junctions rather than a single junction. During the time between tunneling events across an intergrain junction, there can be a redistribution of the charge onto neighboring grains. Such charge fluctuations will tend to increase the observed capacitance because the area probed by the electron is greater $[22]^{\# 3}$. The net result will be a decrease in the charging energy, yielding an effective value, $E_{\mathrm{C}}^{\text {eff }}$. This process will be determined by the mobility of electrons on the network and thus should be more pronounced when the intergrain tunnel resistance $\left(R_{t}\right)$ is low. A similar picture might also be relevant for a single junction. As suggested in ref. [19], for low $R_{\mathrm{t}}$ the tunneling rate may be sufficiently high for an electron to tunnel back and forth several times during the interval in which it contributes to transport. It is possible that the mobility which determines the charge redistribution in our films is temperature dependent. This would imply a $T$-dependent $E_{\mathrm{C}}^{\text {eff }}$, which may be pertinent to the thermopower data. These ideas require further theoretical support. We now discuss magnetoresistance measurements which suggests that the average intergrain tunnel resistance in our films increases with oxidation and with decreasing temperature.

For $R_{\square}(4.2 \mathrm{~K})<8 \mathrm{k} \Omega$ these films exhibit the same weak localization magnetoresistance (MR) that is predicted [23] and measured [11,9,24] for homogeneous $\mathrm{Bi}$ films. The phase breaking length at $T=1$ $\mathrm{K}$ is $L_{\varphi} \approx 1000 \AA$. For $H>H_{\varphi} \equiv \phi_{0} / 4 \pi L_{\varphi}^{2}$ ( $\phi_{0}$ is the flux quantum) and in the presence of strong spin-

\#2 The temperature dependence of the thermopower depends on the relationship between the time of tunneling and the characteristic relaxation time of the electrons within a grain. This issue will be discussed in more detail in ref. [21].

\#3 The authors of ref. [22] find, in a theoretical study, that the effective charging energy of a coupled one-dimensional array of junctions is reduced below that of a single junction if electrons on neighboring junctions can redistribute during the time of tunneling. 
orbit scattering (antilocalization) we have

$\frac{\Delta R}{R}=\frac{R(H)-R(0)}{R(0)}=\frac{e^{2}}{4 \pi^{2} \hbar} r_{\square} \ln H$,

where $r_{\square}$ is the sheet resistance of a homogeneous film. The MR for $R_{\square}(4.2 \mathrm{~K})=38.1 \mathrm{k} \Omega$, shown at several temperatures in fig. 3 , has a dependence that is qualitatively similar to weak localization, behaving as $\ln H$ at high fields and $H^{2}$ at low fields. However, fitting to eq. (1) yields $r_{\square} \ll R_{\square}$. This is to be expected since $R_{\square}$ reflects percolation effects whereas $r_{\square}$ is a measure of the sheet resistance on the scale of the Landau orbit radius, $L_{\mathrm{H}}$. This is the characteristic diffusion length which cuts off the coherent backscattering responsible for weak localization in a field $H>H_{\omega}$. In the logarithmic field regime this length is smaller than the average grain diameter for our films. The following arguments lead us to conclude that $r_{\square}$ measures the average intergrain tunnel resistance for highly oxidized films. Consider two points within a grain, one at the center and the other near the junction with a neighboring grain. The contribution at each such point to the $\ln H$ magnetoresistance is determined by summing the backscattering amplitudes associated with elastic scattering

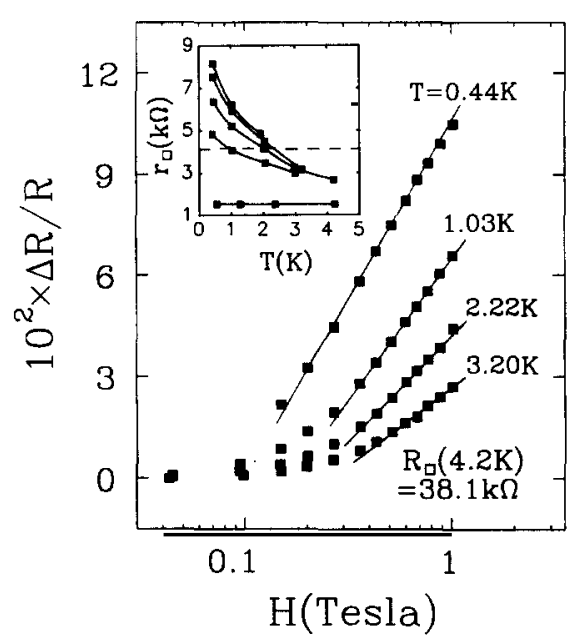

Fig. 3. Magnetoresistance at several temperatures for the oxidized film with $R_{\square}(4.2 \mathrm{~K})=38.1 \mathrm{k} \Omega$. The inset shows $r_{\square}$ versus $T$ for different oxidation levels as determined from the magnetoresistance data (see text). Values of $R_{\square}(4.2 \mathrm{~K}$ ) are, from bottom to top (in $\mathrm{k} \Omega$ ): $1.47,8.36,18.0,38.1,62.6$. The dashed line is $r_{\square}=\hbar e^{2} \approx 4.12 \mathrm{k} \Omega$. "trajectories" [25] (and their time-reversed contours), each of which begin and end at that point and have lengths less than $L_{H}$. For the point at the center of the grain all relevant trajectories are within the grain and hence the MR is the same as that for a homogeneous film with a sheet resistance equal to that of the weakly disordered grains (i.e. $<1 \mathrm{k} \Omega$ ). For the point near the junction we must consider trajectories which cross the junction and return. As long as these trajectories are statistically independent the contribution to the MR will have the same form as for the center of the grain, but will be weighted by the intergrain tunneling resistance $R_{\mathrm{t}}$. When the latter is much larger than the intragrain sheet resistance, as in our highly oxidized films, the overall prefactor of the total MR is predominantly determined by $R_{t}$.

The inset in fig. 3 shows $r_{\square}(T)$, as determined using eq. (1), for different levels of oxidation. A most remarkable observation is that, for each level of oxidation, $r_{\square}$ crosses $\hbar / e^{2}$ at approximately the same temperature below which the thermopower data deviate from metallic ( $T$-linear) behavior. This is consistent with our suggestion that $E_{C}^{\text {eff }}$ depends on temperature through $R_{\mathrm{t}}(T)$. Extending this idea further, in fig. 4 we relate the magnetoresistance and thermopower data by plotting $E_{\mathrm{C}}$ versus $r_{\square}$. We first extract the dependence of $E_{\mathrm{C}}$ on temperature by assuming that the thermopower is given by $S \propto E_{\mathrm{C}}^{\text {eff }} /$ $k_{\mathrm{B}} T$. We draw $1 / T$ curves through each of the $S(T)$ data points at temperatures below the crossover and $E_{C}^{\text {eff }}$ is then obtained from the slope. We next determine $E_{\mathrm{C}}\left(r_{\square}\right)$ by interpolating from the $r_{\square}(T)$ data (fig. 3, inset). The universal sharp transition in $E_{\mathrm{C}}$ versus $r_{\square}$ for both different oxidation and temperatures may reflect a fundamental interplay between Coulomb interaction effects and localization in granular metals. This poses a stimulating theoretical problem for future investigation.

We conclude with some general comments. We expect $E_{\mathrm{C}}^{\text {eff }}$ to saturate at its geometric value $\left(E_{\mathrm{C}}\right)$ when $R_{\mathrm{t}}>10 \mathrm{k} \Omega$, and presumably this occurs at temperatures somewhat lower than $0.2 \mathrm{~K}$ for the intermediate levels of oxidation. Thus in the $T \rightarrow 0$ limit we expect the thermopower for all levels of oxidation to behave as $S \propto E_{\mathrm{C}} / k_{\mathrm{B}} T$; the curves should not cross. The tendency of the curves to merge at the lowest temperatures suggests that the average geometric ca- 


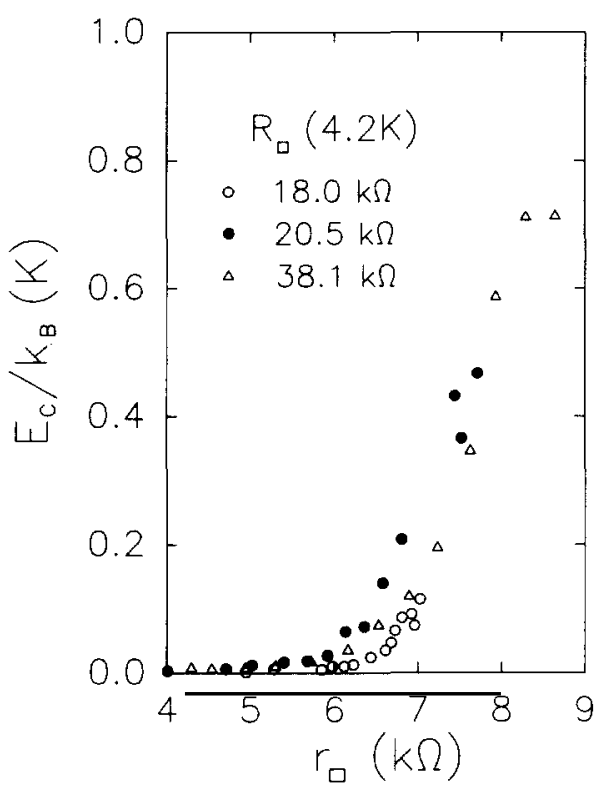

Fig. 4. The charging energy $E_{\mathrm{C}}$, as determined from the thermopower data, plotted versus $r_{\square}$ for three levels of oxidation (values of $R_{\square}(4.2 \mathrm{~K})$ ).

pacitance does not change substantially with oxidation. As mentioned in ref. [12], the third law of thermodynamics requires that the ratio of the thermopower to the resistance tend to zero as $T \rightarrow 0$. Thus we anticipate that the resistance will take on a stronger temperature dependence (probably exponential) when $R_{\mathrm{t}} \gg 10 \mathrm{k} \Omega$. It would be of great interest to test these observations in other granular metal films and in fabricated tunnel-junction arrays.

The authors have benefitted from fruitful discussions with $\mathrm{M}$. Amman and $\mathrm{Y}$. Gefen, and gratefully acknowledge the comments of F. Guinea, K. Mullen, B. Orr, U. Sivan, and Y. Shapir. This work was supported, in part, by National Science Foundation, Low Temperature Physics Grant No. DMR-8508392, NSF Grant No. DMR-8608305 and Grant DAAL 03-87k-0007.

\section{References}

[1] R.C. Dynes, J.P. Garno and J.M. Rowell, Phys. Rev. Lett. 40 (1978) 479;

G. Deutscher, B. Bandyopadhyay, T. Chui, P. Lindenfeld, W.L. McLean and T. Worthington, Phys. Rev. Lett. 44 (1980) 1150 ;

T. Chui, G. Deutscher, P. Lindenfeld and W.L. McLean, Phys. Rev. B 23 (1981) 6172.

114
[2] P. Abeles, P. Sheng, M.D. Coutts and Y. Arie, Adv. Phys. 24 (1975) 407.

[3] E. Abrahams, P.W. Anderson, D.C. Licciardello and T.V. Ramakrishnan, Phys. Rev. Lett. 42 (1979) 673.

[4] A.L. Efros and M. Pollak, eds., Electron-electron interactions in disordered systems (North-Holland, Amsterdam, 1985).

[5] P. Sheng, B. Abeles and Y. Arie, Phys. Rev. Lett. 31 (1973) 44; P. Sheng and J. Klafter, Phys. Rev. B 27 ( 1983) 2583.

[6] A.L. Efros and B.I. Shklovskii, J. Phys. C 8 (1975) L49; O. Entin-Wohlman, Y. Gefen and Y. Shapira, J. Phys. C 16 (1983) 1161.

[7] Z. Ovadyahu and J. Imry, J. Phys. C 16 (1983) 1471; 18 (1985) L19;

B.I. Beletsev, Yu.F. Komnik and A.V. Fomin, Sov. Phys. Solid State 28 (1986) 1139.

[8] J.L. Cohn and C. Uher, J. Appl. Phys. 66 (1989) 2045.

[9] J.L. Cohn, PhD thesis, University of Michigan (1989).

[10] P.A. Lee and T.V. Ramakrishnan, Rev. Mod. Phys. 57 (1985) 287.

[11] Yu.F. Komnik, E.I. Bukhshtab, A.V. Butenko and V.V. Andrievskii, Solid State Commun. 44 (1982) 865;

P.H. Woerlee, G.C. Verkade and A.G.M. Jansen, J. Phys. C 16 (1983) 3011;

F. Komori, S. Koboyashi and W. Sasaki, J. Phys. Soc. Japan 52 (1983) 368.

[12] F.J. Blatt, P.A. Schroeder, C.L. Foiles and D. Greig, Thermoelectric power of metals (Plenum, New York, 1976).

[13] J.M. Ziman, Electrons and phonons (Clarendon, Oxford, 1960).

[14] M.J. Bums, W.C. McGinnis, R.W. Simon, G. Deutscher and P.M. Chaikin, Phys. Rev. Lett. 47 (1981) 1620; M.J. Burns and P.M. Chaikin, Phys. Rev. B 27 (1983) 5924.

[15] D.V. Averin and K.K. Likharev, J. Low Temp. Phys. 62 (1986) 345;

K. Mullen, E. Ben-Jacob, R.C. Jaklevic and Z. Schuss, Phys. Rev. B 37 (1988) 98;

E. Ben-Jacob, Y. Gefen, K. Mullen and Z. Schuss, Phys. Rev. B 37 (1988) 7400 .

[16] T.A. Fulton and G.J. Dolan, Phys. Rev. Lett. 59 (1987) 109; B. Barner and S.T. Ruggiero, Phys. Rev. Lett. 59 (1987) 807;

P.J.M. van Bentum, H. van Kempen, L.E.C. van de Leemput and P.A.A. Teunissen, Phys. Rev. Lett. 60 (1988) 369.

[17] D.J. Thouless, Phys. Rev. Lett. 39 (1977) 1167.

[18] R. Brown and E. Simanek, Phys. Rev. B 34 (1986) 2957.

[19] L.J. Geerligs, V.F. Anderegg, C.A. van der Jeugd, J.R. Romijn and J.E. Mooij, preprint (1989).

[20] A.J. McGeown and C.J. Adkins, J. Phys. C 19 (1986) 1753.

[21] J.L. Cohn, M. Amman, E. Ben-Jacob and C. Uher, unpublished.

[22] M. Amman, E. Ben-Jacob and K. Mullen, unpublished (1989).

[23] S. Hikami, A.I. Larkin and Y. Nagaoka, Prog. Theor. Phys. 63 (1980) 707.

[24] J.L. Cohn, E. Ben-Jacob and C. Uher, unpublished.

[25] A.I. Larkin and D.E. Khmelnitskii, Sov. Phys. Usp. 25 (1982) 185. 